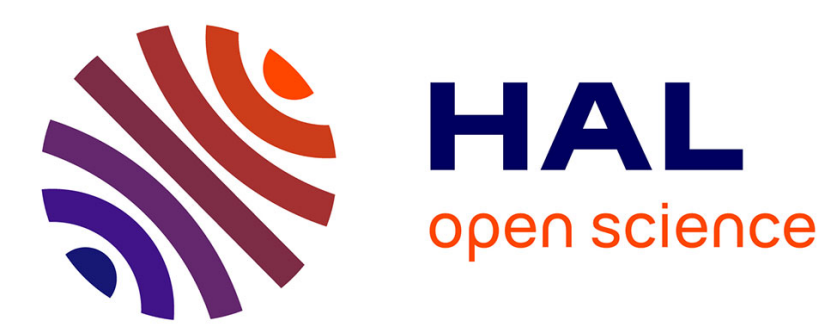

\title{
Design of a Customized Pattern for Improving Color Constancy Across Camera and Illumination Changes
}

Hazem Wannous, Sylvie Treuillet, Yves Lucas, Alamin Mansouri, Yvon Voisin

\section{To cite this version:}

Hazem Wannous, Sylvie Treuillet, Yves Lucas, Alamin Mansouri, Yvon Voisin. Design of a Customized Pattern for Improving Color Constancy Across Camera and Illumination Changes. VISAPP 2010 Fifth International Conference on Computer Vision Theory and Applications, May 2010, Angers, France. pp.60-67. hal-00648510

\section{HAL Id: hal-00648510 https://hal.science/hal-00648510}

Submitted on 5 Dec 2011

HAL is a multi-disciplinary open access archive for the deposit and dissemination of scientific research documents, whether they are published or not. The documents may come from teaching and research institutions in France or abroad, or from public or private research centers.
L'archive ouverte pluridisciplinaire HAL, est destinée au dépôt et à la diffusion de documents scientifiques de niveau recherche, publiés ou non, émanant des établissements d'enseignement et de recherche français ou étrangers, des laboratoires publics ou privés. 


\title{
DESIGN OF A CUSTOMIZED PATTERN FOR IMPROVING COLOR CONSTANCY ACROSS CAMERA AND ILLUMINATION CHANGES
}

\author{
Hazem Wannous \\ IMS Laboratory, University of Bordeaux, Talence, France \\ hazem.wannous@ims-bordeaux.fr \\ Sylvie Treuillet, Yves Lucas \\ PRISME Institute, University of Orleans, Orleans, France \\ sylvie.treuillet@univ-orleans.fr,yves.lucas@bourges.univ-orleans.fr \\ Alamin Mansouri, Yvon Voisin \\ Le2i Laboratory, University of Burgundy, Auxerre, France \\ alamin.mansouri@u-bourgogne.fr,yvon.voisin@u-bourgogne.fr
}

Keywords: Color imaging, color checker design, chromatic adaptation.

\begin{abstract}
This paper adresses the problem of color constancy on a large image database acquired with varying digital cameras and lighting conditions. Automatic white balance control proposed by an available commercial camera is not sufficient to provide reproducible color classification. A device-independent color representation may be obtained by applying a chromatic adaptation transform, from a calibrated color checker pattern included in the field of view. Instead of using the standard Macbeth color checker, we suggest to select judicious colors to design a customized pattern from contextual information. A comparative study demonstrates that this approach insures a stronger constancy of the interesting colors before the vision control.
\end{abstract}

\section{INTRODUCTION}

The human visual system ensures color constancy, so that the perceived color of objects remains relatively constant under varying illumination conditions. This ability, described by the retinex theory, involves both the eye and the brain (Land, 1977). But no digital camera has this ability and color coordinates of pixels are highly depending on acquisition conditions and camera tuning (Barnard and Funt, 2002). Color constancy generally addresses the illumination changes, also called white balancing (Barnard et al., 2002). But, a second problem is caused by changing the camera. While each digital camera provides a device-dependent RGB color coordinates system and embeds a custom color adjustment processing, a more complete chromatic adaptation transform is required for minimizing the color differences between several cameras. The radiometric response function of the camera is generally non linear, different for each color channel and depends on exposure settings such as aperture, focal length and shutter speed. In addition, each commercial digital camera embeds some hidden secret color processing like color demosaicing, white balance adjustment and illumination color correction automatically applied in JPEG format. So, some precautions have to be taken with commercial digital cameras to ensure a consistent color analysis from JPEG images.

In the case of our medical application (Wannous et al., 2007; Wannous et al., 2008), images are acquired in a free manner in several care centers with different types of cameras. In routine, medical staff have no time and no technical competence for using RAW format. But, color constancy is the main cue for a reliable monitoring of tissue classification over time. If a scene was recorded by a free handled camera under several view points, there would be color variations between images of the same object. In that case color constancy may be improved by radiometric alignment between images.

In some previous works algorithms are proposed for estimating the radiometric response function from differently illuminated images taken with the same camera (Debevec and Jitendra, 1997; Mitsunaga and Nayar, 1999; Grossberg and Nayar, 2002; Kim and Pollefeys, 2004). Then, the color textures of images may be aligned by the estimated function. Most of these methods require prior knowledge of exposures on a static scene observed with a fixed camera (Debevec and Jitendra, 1997; Mitsunaga and Nayar, 1999). Extensions have been proposed for non static scene (Grossberg and Nayar, 2002) and free movement of the camera or adding vignetting correction 
(Kim and Pollefeys, 2008). Such a radiometric alignment addresses images acquired with a single digital camera but is not adapted to a large image dataset acquired over time in several medical centers with different commercial digital cameras.

Indeed, automatic white balancing and color adjustments embedded in digital cameras do not ensure color constancy as the applied algorithms differs from a trademark to another. Each digital camera provides a device-dependent RGB color coordinates system. So, different cameras can exhibit radically different color responses and can cause significant errors in scene interpretation (Ilie and Welch, 2005). Furthermore, digital cameras - even of the same type - do not give consistent response. So, we need to homogenize the photos of the data base before the classifier learning stage. A reasonable consistency can be obtained by pair-wise correlation for modeling transfer function based on image color histogram (Porikli, 2003). But the complexity of this approach increases quadratically with the number of cameras and may introduce distortions and quantization errors

In this context, a good solution to achieve a color alignment between images captured with different cameras is given by introducing a small calibrated color pattern in the field of view during acquisition. Then, color values may be converted in a deviceindependent coordinate system by estimating the best adaptation transform that maps the image color measurements to the corresponding target reference coordinates. This on-line calibration process reduces the color variations due to illumination and camera changes and consequently ensures the reproducibility of the automatic segmentation and classification of the textured color regions. A classical choice for the color pattern is the 24-sample Macbeth color checker (Barnard et al., 2002; Haeghen et al., 2000; Ilie and Welch, 2005; Mansouri et al., 2005). This standard pattern consists of 24 patches chosen to emulate common natural colors such as skin, foliage, and sky, in addition to primary colors and a six step grey scale. It covers an extensive gamut, adapted to a large range of images. But a common drawback of a calibration with such a standard pattern is the decrease in performance of constancy when interesting colors are not represented in the color checker. In fact, many surface inspection problems are based on a limited palette of colors. Then, a good strategy for color calibration would be to minimize the mean square errors in some judiciously selected areas of the color space, to ensure stronger constancy of these interesting colors.

In this paper, we propose a methodology for extracting judicious colors to design a customized pattern by analyzing contextual information in a large image dataset. This issue deals with the color quantization problem for automatically extracting dominant colors in images by some adaptive clustering algorithm (Hsieh and Fan, 2000; Cheng and Yang, 2001; Sirisathitkul et al., 2004). But here, the aim is not to classify pixels into their corresponding palette colors, but to design a specific color chart. So, the dominant colors were selected from a set of images instead of a single image. Next, a special sorting algorithm was developed to reduce these colors to a very small set while saving the best share-out in the color space. A comparative study between correction results obtained with the standard Macbeth color checker chart and our customized one proves that the color distance errors are minimized, to consequently provide a more robust automatic classification in the area of interest.

The organization of the paper is as follows: in the next section, we present the proposed methodology for designing a contextual checker chart. The adopted correction process for insuring color constancy across camera and illumination changes is detailed in section 3 . In section 4, we present the results of the proposed approach before to conclude in the last section.

\section{DESIGN OF A CUSTOMIZED COLOR CHECKER}

Considering a given surface inspection problem, the color consistency has to be particularly centered on a limited palette of interesting colors. In this section, we propose an automatic method to customize a color checker pattern using contextual data scene statistics.

The first thing to do is to collect a large dataset of images under varying illuminations and cameras in real conditions relative to the aimed application. All the images have been captured with a small standard Macbeth color checker pattern included in the field of view, to be first normalized by applying a standard color calibration detailed in the next section.

To reduce the combinatorial complexity of the 24-bit color representation, we apply a perceptual color quantization for automatically extracting dominant colors in this collection of images. The preprocessing stage includes a Peer group filtering algorithm to remove impulsive noise in the images and to compute a weighting index used after for color quantization (Deng et al., 1999). Homogeneous neighboring pixels are favored by higher weights. Next, the generalized-Lloyd algorithm (GLA) is applied in the perceptive CIELUV space to drastically reduce the number of colors by clustering. These choices are justified by the use of a similar pre-processing stage during color region segmentation (Deng and Manju- 
nath, 2001). Among several advanced segmentation methods, the JSEG algorithm proved to be the most efficient on our dermatological image database. Nevertheless, this choice can be suited to a large range of applications as a JSEG method includes a reliable color quantization stage. The quantization results in a codebook limited to some dominant colors for each image (Fig. 1).
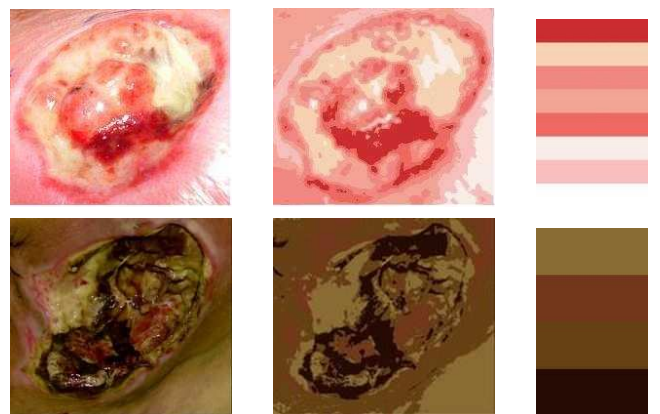

(a)

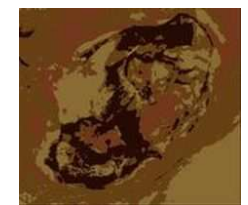

(b)

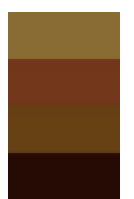

(c)
Figure 1: Color quantization by generalized-Lloyd algorithm (GLA): (a) original image, (b) image quantization (c) color codebook.

We collected 132 colors from a base of 26 images as the most representative ones for our application (Fig. 2).

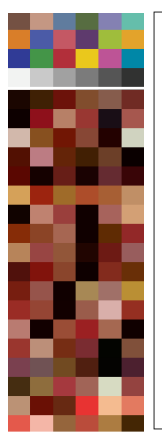

(a)

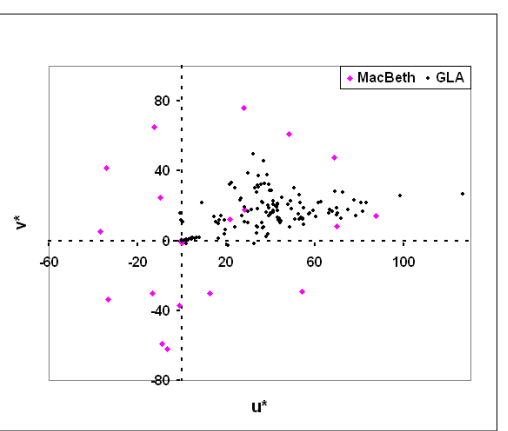

(b)
Figure 2: Codebook of 132 colors selected by GLA on the image data base compared the 24 Macbeth color patches (a) corresponding coordinates in the CIELUV chromaticity plan (b).

As it can be seen on the right part of the Fig. 2, these dominant colors are not uniformly spread in the CIELUV chromaticity plan $\left(\mathrm{u}^{*}, \mathrm{v}^{*}\right)$. They form a stretched cloud of points. A principal component analysis of these data points shows that the principal axis is defined by the unit vector [0.97; 0.24]. This axis of the largest variance (explicated inertia of 0.87 ) is highly correlated to the $\mathrm{u}^{*}$ axis. This observation agrees with the examination of the colors of skin tissues which present hue gradation from pink to beefy red, yellow and brown. By comparison, the gamut covered by the Macbeth color checker is really extensive, but only five patches of the Macbeth pattern are confined in the area corresponding to the dominant colors selected by GLA. So, the aim is to select about twenty judicious colors among the dominant ones to design a customized pattern. The proposed selection algorithm is based on two basic ideas: give greater importance to the colors with the highest occurrences while preserving the spreading in the chromaticity plan. The proposed approach for sorting the most representative colors is the following iterative algorithm.

Algorithm: Color checker design using $\Delta E_{u v}$ distance between candidate colors.

Input: a data set of candidate colors $\mathrm{D}=\left\{L_{d}, u_{d}, v_{d}, \mathrm{~d}=\right.$ $1,2, \ldots, \max \}$ and a predefined $K$ number of representative colors to be selected).

Output: a $K$-color codebook $P=\{(L p, u p, v p), p=$ $1,2, \ldots, K\}$

Method:

1. keep all the candidate colors for $u *$ and $v *$ values in range $\mp 100$

2. compute the $\Delta E_{u v}$ distance between all pairs of the candidate colors

3. sort the candidate colors along the axis of their increasing distance adjacent in an ordered whole. Choose the pair, with the smallest distance $\Delta E_{u v}$, among $\sum_{i=1}^{N}(N-i)$ pairs of $N$ colors as origin of this axis. Let $D_{j}=d^{2}\left(c_{j}, c_{j-1}\right)$ be the squared Euclidian distance of adjacent colors in CIELUV space

4. divide this whole of colors into $(L<K)$ consecutive cells where each cell contains the same number of colors

5. for of each cell, compute the summation of distances between the adjacent colors $d_{s u m_{i}}=\sum_{j=1}^{i}\left(D_{j}\right)$ 6. if $\mathrm{dsum}_{i} \geq \tau$ collect the median color in each cell to produce $m$ color codebook, else merge the remaining cells in one and divide the latter in $K-m$ cells where the $d s u m_{i}$ in each one equal to $(N-m) / K$

7. collect the median color in each cell to produce $(K-m)$ color codebook.

8. stop

Step 3 of the algorithm guarantee an extended recovery of the workspace (samples), while step 6 takes into account the distribution of samples (candidate color) in the uniform perceptual space CIELUV.

We note finally that the splitting threshold $\tau$ and the initial number of cells $L$ have been tuned empirically in such a way as to obtain K-color codebook.

This algorithm has been applied to the previous set 
of 132 candidate colors selected by GLA on the image data base, with $\mathrm{K}=22$. This choice enables to obtain a target of similar size than MacBeth. The result is illustrated in Fig.3. Black and white patches are added to the 22 selected colors to design the customized pattern. The latter has the advantage of proposing a large palette of representative colors included in chronic wound images while keeping a compact size. The two color checkers (the standard Macbeth pattern and the customized one) have been printed on a calibrated printer with the coordinates assigned to each patch, and next used in the following experimental tests.

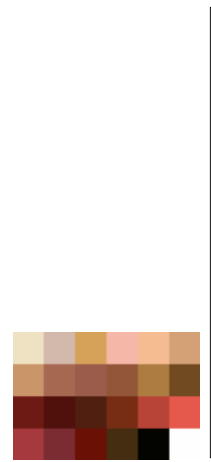

(a)

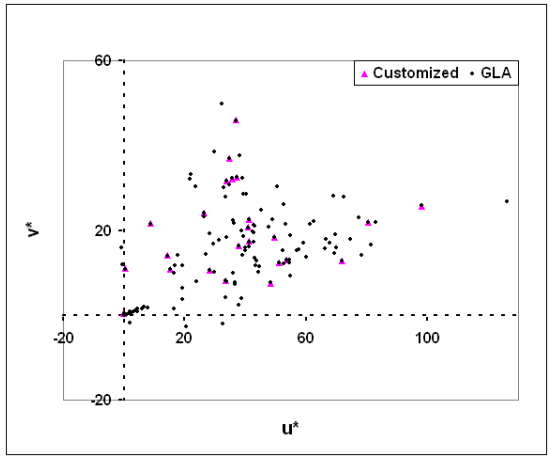

(b)
Figure 3: Customized color checker and the corresponding coordinates in the CIELUV chromaticity plan.

\section{CORRECTION STRATEGY}

Color constancy generally addresses the illumination changes by so-called white balancing, but this does not takes into consideration the changing of the camera. While each digital camera provides a devicedependent RGB color coordinates system and embeds a custom color adjustment processing, a more complete chromatic adaptation transform is required for minimizing the color differences between several cameras. The correction strategy is then composed of two steps.

\subsection{White balancing}

The colorimetric data provided by a digital camera can not be dissociated from the illuminant coordinates. Many algorithms of computer vision have been developed to model color constancy (McCann, 2004) under illumination changes. Most of them adopt retinex models and the assumption of the independence of each color channel. The dependence to illuminant may then be corrected by a $3 \times 3$ linear transform on the RGB coordinates, as follows:

$$
\left[\begin{array}{c}
R_{c} \\
G_{c} \\
B_{c}
\end{array}\right]=\left[\begin{array}{ccc}
R_{m} / R_{W} & 0 & 0 \\
0 & G_{m} / G_{W} & 0 \\
0 & 0 & B_{m} / B_{W}
\end{array}\right] \cdot\left[\begin{array}{c}
R \\
G \\
B
\end{array}\right]
$$

where $\left(R_{W}, G_{W}, B_{W}\right)$ are the white reference coordinates, $\left(R_{m}, G_{m}, B_{m}\right)$ the measured coordinates and $\left(R_{c}, G_{c}, B_{c}\right)$ the corrected ones.

White balancing control is realized by digital image processor embedded in cameras. Digital cameras often propose two modes: automatic or manual white balancing. The manual mode of cameras requires to capture an image of a white paper sheet under the ambient lighting to store the white source coordinates. But this is not a convenient use for a routine visit in a patient room. The automatic white balance control is based on stored reference coordinates of some standard illuminants, which are generally considered as the maximal RGB values present in the image. The ambient lighting in the patient rooms, composed of several varying sources, may be indeed far away from the stored standard illuminants, and because of the centering, the maximal RGB values in the image may not be localized on really white object.

For this reason, the channel ratios applied on the color coordinates of each pixel will be based on the white patch coordinates $\left(R_{m}, G_{m}, B_{m}\right)$ measured on the checker and the reference ones $\left(R_{W}, G_{W}, B_{W}\right)$ defined during calibration stage under D65 standard illuminant (CIE, 2008). The above linear correction allows to obtain white balanced images whatever the lighting conditions.

\subsection{Chromatic adaptation transform}

In order to ensure the color constancy across multiple cameras, we apply an on-line calibration process based on the known color target included in the field of view. This calibration allows converting the measured color values in a device-independent coordinates system (as in SRGB space) by estimating the following transform $\Phi$ with $m$ terms. It can be written as:

$$
\begin{aligned}
\left(\begin{array}{c}
R_{c} \\
G_{c} \\
B_{c}
\end{array}\right) & =\Phi_{R G B \rightarrow s R G B}\left(\begin{array}{c}
R \\
G \\
B
\end{array}\right) \\
& =\left(\begin{array}{lll}
a_{1} & \cdots & a_{m} \\
b_{1} & \cdots & b_{m} \\
c_{1} & \cdots & c_{m}
\end{array}\right)\left[\Theta_{m}\left(\begin{array}{c}
R \\
G \\
B
\end{array}\right)\right]
\end{aligned}
$$

Our transformation is based on a polynomial model. The transformation coefficients are computed 
by the closest match between the reference coordinates of the target patches and the measured coordinates in the images. The reference coordinates of the 24 color patches of the target are provided by a spectrophotometer (Minolta CS 1000 SPM), under the standardized D65 illuminant for the $10^{\circ}$ field of view.

The best chromatic adaptation was obtained using the lower order $(m=3)$ which is in good agreement with other works (Haeghen et al., 2000). More general polynomial functions have also been tested but they do not give significant enhancement while they cause largest distortions and higher computation complexity. Each reference color patch provides 3 equations, allowing to estimate 3 by $\mathrm{N}$ parameters for $\mathrm{N}$ patches. With lower order and 22 patches, the linear system is over determined and a least mean squares solution is provided.

Even if the standard calibration with Macbeth stabilizes the segmentation and classification steps, as illustrated in Fig.4, it can be seen that some interesting colors for medical diagnosis are not optimally corrected. That can be explained by the fact that the minimization of errors is constrained only at some selected points of the color space represented on the pattern. A better strategy would be to use a customized checker by selecting judicious colors to insure the closest constancy of these interesting colors, as presented in the next section.

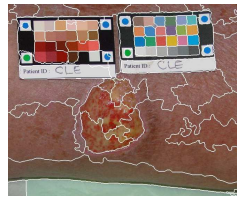

(a)

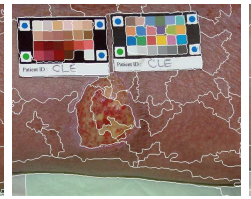

(b)

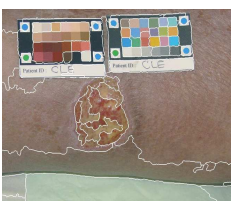

(c)
Figure 4: Segmentation of a wound image captured under automatic white balance control $(a)$ and after color correction using the calibrated color checker included in the field of view: $(b)$ Macbeth pattern $(c)$ customized pattern.

\section{EXPERIMENTAL RESULTS}

In this section, we show the effectiveness of the proposed correction by several experiments. We use two sets of real images: the first is composed of images taken under controlled lighting conditions in laboratory; the second one includes in vivo images taken in hospital environment. The Macbeth and the customized color charts have been calibrated with the same spectrophotometer.
The location of the color checker is automatically detected in the images, and the checker is replaced in a facial position by applying a robust homographic transform to remove the perspective effects. Hence the choice of a specific shape of the color checker target with four colored balls facilitates automatic detection. The average RGB coordinates are then extracted in the squared area centered in each patch.

The color correction is generally applied in sRGB space for its convergence with the PC-world, but to make quantitative evaluation, we must use a perceptually uniform space such as CIELAB. The individual inter-sample deviation is computed by the Euclidian distance between the measured coordinates and the reference ones in CIELAB space for each patch. Then, we take the average color difference of the 24 patches to measure the differences between the images and the reference ones.

\subsection{Measures under controlled illumination}

For comparison purpose, Macbeth and customized targets have been jointly placed in a lighting test box. Images has been captured under three different controlled lighting: a cool fluorescent light (Illuminant F - $4150 \mathrm{~K}$ ), an incandescent light (Illuminant A $2856 \mathrm{~K}$ ), and a daylight (Illuminant D65 - $6500 \mathrm{~K}$ ). Two different models of cameras have been tested (canon EOS 350D, Leica D-Lux 3). The images were stored in RAW format to avoid embedded custom color processing and next to be compared to JPEG compressed images. The scene has been observed from five different points of view: a centering front view and four cardinal views with angle about $25^{\circ}$, corresponding to our clinical protocol for 3D reconstruction (?). Each view has been repeated 5 times, i.e. a total of 600 measures ( 24 patches x 5 views x 5 acquisitions) to compute the average $C I E \Delta E_{a b}$ distance for each camera and each illuminant, before and after correction. Results are presented in Table 1.

CIELAB color distances are measured simultaneously on the Macbeth color checker and on the customized pattern before and after correction. The 'standard correction' indicates that the chromatic adaptation is computed from the MacBeth pattern; the 'proposed correction' corresponds to a chromatic adaptation computed from the customized pattern.

Before correction, we observed very large differences between the two types of digital cameras under different illuminants, with an average around 25 units. A smaller standard deviation has also been observed on the customized pattern, which may be explained 


\begin{tabular}{cccccccc}
\hline CIELAB distances & illuminant & \multicolumn{3}{c}{ Canon EOS 350D } & \multicolumn{3}{c}{ Leica D-Lux 3 } \\
\cline { 2 - 8 } & & Max & Average & Std & Max & Average & Std \\
\hline Macbeth target & Cool & 39.78 & 23.35 & 4.18 & 51.13 & 23.11 & 1.39 \\
before correction & A & 36.17 & 26.82 & 2.19 & 48.78 & 21.45 & 1.59 \\
& D65 & 39.50 & 28.32 & 2.21 & 46.59 & 24.57 & 4.44 \\
& & & & & & & \\
Customized target & Cool & 31.40 & 22.82 & 3.26 & 21.08 & 14.28 & 0.84 \\
before correction & A & 32.34 & 26.21 & 1.44 & 23.69 & 14.12 & 1.14 \\
& D65 & 36.80 & 28.93 & 1.64 & 45.21 & 16.52 & 4.04 \\
Macbeth target & Cool & 8.45 & 3.94 & 0.12 & 9.89 & 3.04 & 0.30 \\
standard correction & A & 8.97 & 4.01 & 0.13 & 11.05 & 3.36 & 0.41 \\
& D65 & 7.57 & 3.88 & 0.14 & 7.89 & 3.26 & 0.20 \\
customized target & & & & & & & \\
standard correction & Cool & 15.67 & 5.39 & 0.24 & 22.41 & 5.25 & 0.58 \\
& D & 13.51 & 5.22 & 0.29 & 20.01 & 5.15 & 0.54 \\
customized target & Cool & 12.71 & 5.24 & 0.33 & 17.97 & 6.05 & 0.35 \\
proposed correction & A & 5.87 & 2.76 & 0.22 & 5.40 & 2.00 & 0.26 \\
& D65 & 8.46 & 2.86 & 0.13 & 10.15 & 2.04 & 0.36 \\
& 2.94 & 0.17 & 10.37 & 1.90 & 0.23 \\
\hline \hline
\end{tabular}

Table 1: CIELAB color distances measured on the 24 patches after correction with the standard Macbeth color checker and the customized designed one under controlled illumination.

by a more restricted range of colors.

The correction based on the Macbeth pattern reduces the average $C I E \Delta E_{a b}$ by a factor between 5 to 6 , reducing the gap to about 3,5-4. But, for the interesting colors on the customized pattern, the color constancy is not sufficient: the differences remain high with an average around 5-6 and some maximum values between 12 to 22 . Logically, the color constancy is clearly improved by the proposed correction on the customized pattern, as the minimization of errors is constrained on judicious colors confined in the interesting area of the color space.

A second test concerns the influence on the results of color processing and JPEG compression carried out by the digital camera (see Table 2). The JPEG compressing format is more convenient for storing a large image data base, but it implies no control on the embedded color processing, such as demosaicing or color white balancing specific to each camera type. However, the test proves that the correction performances are not significantly modified by all these transformations.

\subsection{In vivo images}

To complete these laboratory experiments, we analysed in a similar way color shifts on photos taken during wound patient examination in several hospitals.
To evaluate the proposed correction in real conditions, the same tests have been applied to a collection of in vivo images taken in hospital environment (see Table 3 ). It confirms that the contextual color correction on the customized pattern results in a higher degree of constancy for interesting colors. In last analysis, we can evaluate the real impact of the color correction at the end of the processing chain: the performance of the classification of the skin tissues is improved and a better agreement is obtained with the medical expert (about $15 \%$ on classification rate) when including the correction step (Wannous et al., 2007).

\section{CONCLUSION}

This paper deals with color constancy on a large image database acquired with varying digital cameras and lighting conditions. Automatic white balance control proposed by the commercial cameras is not sufficient to provide reproducible surface inspection or classification results. The instability in color rendering may be significantly reduced by applying a color calibration with a color checker pattern included in the field of view. A device-independent color representation may then be obtained by applying a chromatic adaptation transform, without the necessity of re-calibrating the cameras when the lighting condi- 


\begin{tabular}{cccccccc}
\hline \hline CIELAB distances & illuminant & \multicolumn{3}{c}{ JPEG format } & \multicolumn{3}{c}{ RAW format } \\
\cline { 2 - 8 } & & Max & Average & Std & Max & Average & Std \\
\hline \multirow{3}{*}{ before correction } & Cool & 25.32 & 16.83 & 1.57 & 21.08 & 14.28 & 1.84 \\
& A & 23.98 & 17.93 & 1.00 & 23.69 & 14.12 & 1.14 \\
& D65 & 41.99 & 21.21 & 3.18 & 45.21 & 16.51 & 4.09 \\
correction & & & & & & & \\
with & Cool & 6.95 & 2.68 & 0.34 & 5.40 & 2.00 & 0.26 \\
customized target & D & 7.19 & 2.73 & 0.44 & 10.15 & 2.04 & 0.36 \\
\hline \hline
\end{tabular}

Table 2: CIELAB color distances measured on the 24 patches of the customized color checker, in JPEG and RAW format.

\begin{tabular}{cccc}
\hline \hline CIELAB distances & \multicolumn{3}{c}{ in vivo images (JPEG) } \\
\cline { 2 - 4 } & Max & Average & Std \\
\hline Macbeth before correction & 29.28 & 15.07 & 6.56 \\
customized before correction & 34.82 & 20.02 & 4.05 \\
Macbeth standard correction & 9.74 & 4.24 & 0.25 \\
customized standard correction & 27.88 & 10.68 & 1.74 \\
customized proposed correction & 8.47 & 3.37 & 0.47 \\
\hline \hline
\end{tabular}

Table 3: CIELAB color distances measured on the 24 patches of the standard Macbeth color checker and the customized one designed for in vivo images.

tions change. Instead of using the standard Macbeth color checker, we suggest to select judicious colors to design a customized chart using contextual information. A comparative study demonstrates that this approach insures a closest constancy of the interesting colors for the vision control. We conclude that using heterogeneous cameras and varying ambient illumination in patient room is possible for computer aided diagnosis of skin lesions. While designed for optimum color constancy in cutaneous imaging, the proposed approach to customize a color checker can be applied to many surface inspection problems requiring a limited palette of colors. Future works will be centered on the adaptation of the number of patches inside the color pattern to the quality of the desired precision.

\section{REFERENCES}

Barnard, K. and Funt, B. (2002). Camera characterization for color research. Color Research and Application, 27(3):153-164.

Barnard, K., Martin, L., Coath, A., and Funt, B. (2002). A comparison of computational colour constancy algorithms, part ii : Experiments with image data. IEEE Trans. on Image Processing, 11(9):985-999.

Cheng, S. and Yang, C. (2001). A fast and novel technique for color quantization using reduction of color space dimensionality. Pattern Recognition Letters, 22 (8):845-856.

CIE (2008). Colorimetry part 2: Standard illuminants for colorimetry. Technical report, Joint ISO/CIE Standard ISO 11664-2:2008(E)/CIE S 014-2/E:2006, CIE Central Bureau, Kegelgasse 27, A-1030 Vienna, Austria.

Debevec, P. E. and Jitendra, M. (1997). Recovering high dynamic range radiance maps from photographs. In Proc. of the 24th annual conference on Computer graphics and interactive techniques, pages 369-378.

Deng, Y., Kenney, S., Moore, M., and Manjunath, B. S. (1999). Peer group filtering and perceptual color image quantization. In IEEE Inter. Symp. on Circ. and Sys. VLSI (ISCAS'99), volume 4, pages 21-24, Orlando, FL.

Deng, Y. and Manjunath, B. S. (2001). Unsupervised segmentation of colour-texture regions in images and video. IEEE Trans. on Pattern Analysis and Machine Intelligence (PAMI '01), 23:800-810.

Grossberg, M. and Nayar, S. (2002). What can be known about the radiometric response function from images ? In Lecture Notes in Computer Sciences (ECCV), volume 2353, pages 393-413.

Haeghen, Y. V., Naeyaert, J. M., Lemahieu, I., and Philips, W. (2000). An imaging system with calibrated color image acquisition for use in dermatology. IEEE Trans. on Medical Imaging, 19(7):722-730.

Hsieh, I.-S. and Fan, K.-C. (2000). An adaptive clustering algorithm for color quantization. Pattern Recognition Letters, 21(4):337-346.

Ilie, A. and Welch, G. (2005). Ensuring color consistency 
across multiple cameras. In IEEE Int. Conf. on Computer Vision, volume 2, pages 1268-1275.

Kim, S. and Pollefeys, M. (2004). Radiometric selfalignement of image sequences. In Int. Conf. on Computer Vision and Pattern Recognition, volume 1, pages 645-651.

Kim, S. and Pollefeys, M. (2008). Robust radiometric calibration and vignetting correction. IEEE Trans. on Pattern Analysis and Machine Intelligence, 30 (4):562576.

Land, E. H. (1977). The retinex theory of color vision. Scientific American, pages 108-128.

Mansouri, A., Marzani, F., and Gouton, P. (2005). Development of a protocol for ccd calibration: application to a multispectral imaging system. IJRA, 20(2):pp. 94100.

McCann, J. (2004). Mechanism of color constancy. In IS\&T/SID Conf. on Color Imaging, volume 12, pages 29-36.

Mitsunaga, T. and Nayar, S. (1999). Radiometric selfcalibration. In Conf. on Vision and Pattern Recognition, volume 2, pages 374-380.

Porikli, F. (2003). Inter-camera color calibration by crosscorrelation model function. In IEEE Int. Conf. on Image Proc, volume 2, pages 133-136.

Sirisathitkul, Y., Auwatanamongkol, S., and Uyyanonvara, B. (2004). Color image quantization using distances between adjacent colors along the color axis with highest color variance. Pattern Recognition Letters, 25(9):1025-1043.

Wannous, H., Lucas, Y., Treuillet, S., and Albouy, B. (2008). A complete $3 \mathrm{~d}$ wound assessment tool for accurate tissue classification and measurement. In Proc. 15th IEEE International Conference on Image Processing ICIP 2008, pages 2928-2931.

Wannous, H., Treuillet, S., and Lucas, Y. (2007). Supervised tissue classification from color images for a complete wound assessment tool. In 29th Inter. Conf. of the IEEE Eng. in Med. and Bio. Soc. EMBS'07, pages 6031-6034. 